\title{
Breakage Mechanism Study of Overhead Ground Wire Under Lighting Stroke Based On Finite Element Analysis
}

\author{
Yunfeng Xia ${ }^{1}$, Fan Kuang ${ }^{1 *}$, Ting Yang ${ }^{1}$, Guanhao Chen ${ }^{1}$, Rui Yang ${ }^{1}$ \\ ${ }^{1}$ Dongguan Power Supply Bureau of Guangdong Power Grid Corporation, Dongguan 523000, China
}

\begin{abstract}
Breakage of overhead transmission lines and distribution lines will lead to severe power outage problems, and clarifying the breakage mechanism of the overhead lines is of great importance and value for the reliability and stability of the power system. Taking overhead ground wire for example, this paper presents its breakage mechanism study under lighting stoke based on finite element analysis. A multi-physics analysis model is built first, which includes the model of contact points. The current density distribution and the temperature rise under the impact of short circuit current are analysed, and contact resistance effect is proved the primary cause for the breakage. Then some major factors that influence the contact resistance which in turn influence the temperature rise are discussed. Based on the discussions, some valuable suggestions are given for the protection of overhead lines.
\end{abstract}

\section{Introduction}

In the operation of overhead transmission lines and distribution lines, breakage of the lines usually occurs and it will lead to power outage. Statistics shows that, most of the breakage occurs at the connection fittings of the overhead lines [1]. Overhead ground wire and its suspension clamp is the most common case. However, the breakage mechanism is still unclear. Clarifying the breakage mechanism of the overhead ground wire is of great importance and value for the reliability and stability of the power system.

When a lighting occurs, flashover may occur on the overhead transmission lines to the ground (the tower or the overhead ground line) [2]. A large short circuit current to earth is possible to occur and flows in the overhead ground wire and its suspension clamp. Under the impact of the short circuit current, the temperature of the ground wire and its suspension clamp will be greatly increased, and this is the possible reason for the breakage.

The rapid temperature rise of the ground wire and its suspension clamp has two main explanations, namely skin effect [3] and contact resistance effect [4]. As the short circuit current is alternating at power frequency, it tends to flow on the surface of the conductor due to the skin effect. It will increase the equivalent resistance of the wire and increase the power loss. The contact resistance arises from imperfect contact surfaces, which is not smooth, but has many spots. When the short circuit current flows through two contact surfaces, it will flow through several spots, not the entire contact surface [5]. This will also lead to an increase of equivalent resistance and power loss.

Many researches have been performed to measure the contact resistance [6-9]. However, measuring the contact resistance can't directly reflect the temperature rise under the impact of the short circuit current, and can't give the direct evidence why the breakage occurs. So, a reasonable method is to directly measure the temperature rise at the contact points. However, as the short circuit time is very short, it is not easy to accurately measure the temperature rise. Even a measuring equipment with high precise is available, the measurement can't be realized as the contact points are in the deep channel and not easy to be accessed.

Luckily, finite element analysis provides an efficient and powerful tool to analyse the current density distribution and temperature rise of the overhead ground wire and its suspension clamp during the short circuit stage. This paper mainly presents the breakage mechanism study of overhead ground wire based on finite element analysis. This paper is organized as follows. Section 2 will briefly introduce the analysis model and corresponding assumptions. The analysis results, including current density distribution and temperature rise of a typical case from the analysis will be presented in Section 3. The contributions to the breakage from skin effect and contact resistance effect are compared, and the contact resistance effect is proved the primary cause why the breakage occurs. Detailed discussion of the contact resistance effect on the breakage will be presented in Section 4, and some valuable suggestions will be also provided. At last, a summary of this paper will be given.

\section{Analysis model}

\subsection{Geometry Model}

Fig. 1 shows the analysis model of overhead ground wire and its suspension clamp. The ground wire is usually made 
of stainless steel and in stranded type. However, it is modelled as several parallel conductors to simplify the analysis, and each has a diameter of $4 \mathrm{~mm}$. To avoid fatigue wear of the wire, a layer of aluminium armour tape is inserted between the wire and the clamp. In the analysis, the aluminium armour tape is simplified as a cylinder with a thickness of $1 \mathrm{~mm}$.

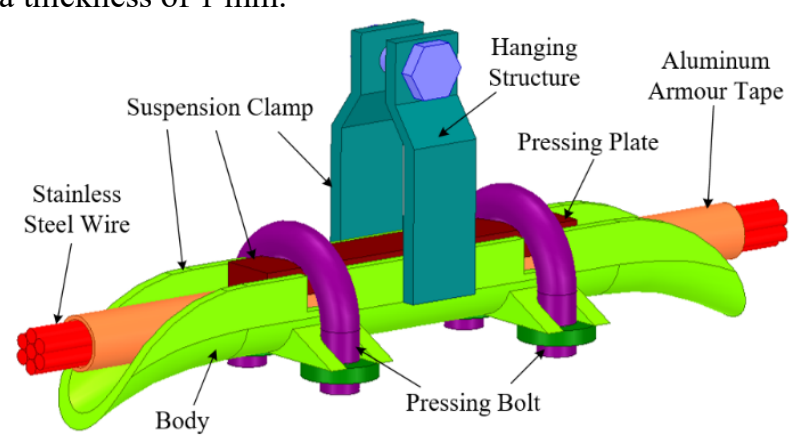

Fig1. Analysis model of overhead ground wire and its suspension clamp

In practice, the aluminum armour tape is used for the protection of the ground wire, not for the electrical connection. So no compression force is required on the aluminum armour tape except for the part in the suspension clamp. In the analysis, the aluminum armour tape is treated as a separation part from the ground wire except for the part compressed by the suspension clamp.

\subsection{Model of Contact Points}

Contact points act a significant role in the breakage of the overhead ground wire under the impact of short circuit current caused by lighting. So, it is necessary to include the effect of the contact points in the analysis. Fig. 2 shows the contact point distribution between the stranded ground wire and the aluminum armour tape. As the ground wire and the aluminum armour tape are compressed by the pressing plate and the body, the contact points mainly distribute on the upper and lower sides.

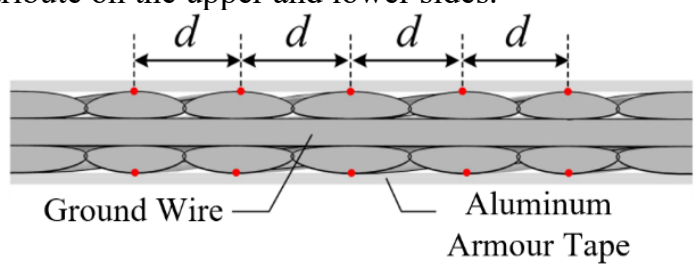

Fig2. The contact point distribution between the ground wire and the aluminum armour tape

If there are $n$ conductors on the outer layer of the wire, there will be $n$ contact points in a lay pitch $L_{\mathrm{p}}$, and the distance between 2 adjacent contact points is $d=L_{\mathrm{p}} / n$ as shown in Fig. 2. In the analysis model shown in Fig. 1, as the ground wire is simplified as several parallel conductors, the contact points are assumed uniformly distributed on the conductors as shown in Fig. 3. For each conductor, there will be one contact point in a lay pitch. In the analysis, the total number of contact points $N_{\mathrm{c}}$ is 60 .
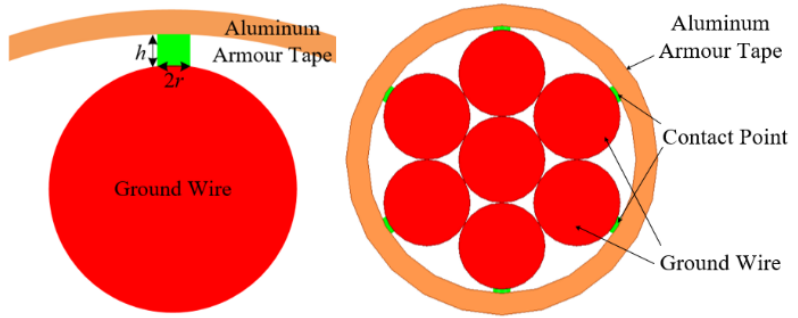

Fig3. Contact point distribution in the analysis model

The effect of contact point is modelled as an electrical bridge with a height of $h$ and a radius of $r$. The material of the electrical bridge is the same as the ground wire. Its height $h$ is set $0.2 \mathrm{~mm}$, and the radius $r$ can be calculated by Holm equation as

$$
r=\sqrt{F_{c} / \pi \xi H N_{c}}
$$

where, $F_{\mathrm{c}}$ is the total compression force on the contact surface, $\xi$ represents the contact condition at the contact surface, $H$ is the Brinell hardness of the material at the contact surface and $N_{\mathrm{c}}$ is the total number of the contact points. The value of $\xi$ usually ranges from 0.3 to 0.6 , and it is selected at 0.5 in the analysis. For the Brinell hardness $H$, it is selected at $180 \mathrm{HB}$.

For the suspension clamp, the compression force can be calculated as

$$
F_{c}=\frac{n T_{c}}{K d_{b}}
$$

where, $n$ is the number of the bolts and it is selected as $4, K$ is the friction coefficient of thread and it is selected as $0.25, d_{\mathrm{b}}$ is the diameter of the bolt and it is selected as $10 \mathrm{~mm}$, and $T_{\mathrm{c}}$ is the torque of the bolt and it is selected as $1 \mathrm{~N} \cdot \mathrm{m}$.

\subsection{Loads and Boundary Conditions}

When a short circuit of a transmission line occurs to the ground, the short circuit current will flow through the tower and the overhead ground line. Previous study shows that, over $80 \%$ percentage of the short circuit current will flow into the ground line, and this is the main reason why the breakage occurs.

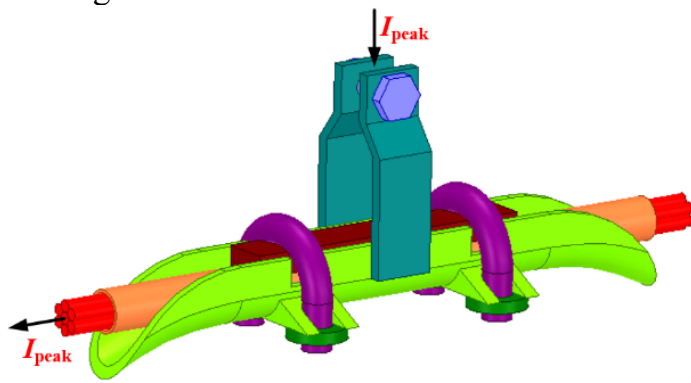

Fig4. Short circuit current loop on the ground wire and the suspension clamp

To evaluate the impact of the short circuit current, current load is applied and its amplitude is assumed as Ipeak. The current is assumed flowing from the hanging structure to one side of the ground wire as shown in Fig. 4. This situation represents the worst case as the current is concentrated on only half of the ground wire and the suspension clamp. In the analysis, the peak value of the short circuit current is assumed $20 \mathrm{kA}$ and its lasting time td is assumed $100 \mathrm{~ms}$. 
When the short circuit current flows through the ground wire and its suspension clamp, especially through the contact points, a large amount of ohmic loss will be generated and give rise to the rapid temperature rise to the conductors. Thus, thermal analysis is also obligatory to evaluate the impact of the short circuit current. Due to the positive temperature coefficient effect, the resistance of the conductors will increase as the temperature rises. So in the analysis, the relation between the resistivity and the temperature is also considered as

$$
\rho_{T}=\rho_{0}\left[1+\alpha\left(T-T_{0}\right)\right]
$$

where, $\rho_{\mathrm{T}}$ is the resistivity at the temperature $T, \rho_{0}$ is the resistivity at the temperature $T_{0}$, and $\alpha$ is the temperature coefficient of resistivity.

As the duration time of the short circuit is very short, the convection and radiation is ignored in the analysis, only the thermal conduction in the conductors is considered. In addition, at the boundary of the analysis model, adiabatic boundary condition is applied.

\section{Analysis results}

\subsection{Current Distribution}

In the electromagnetic analysis, the total short circuit current flowing in the ground wire $I_{\text {peak }}$ is $20 \mathrm{kA}$, the torque of the bolt is $1 \mathrm{~N} \cdot \mathrm{m}$ which corresponds to a radius of 0.3 $\mathrm{mm}$ for the electrical bridges. The conductivity of the ground wire and the suspension clamp is $6 \times 10^{6} \mathrm{~S} / \mathrm{m}$, and the conductivity for the aluminium armour tape is $3.8 \times 10^{7}$ $\mathrm{S} / \mathrm{m}$.

Fig. 5 shows the current density distribution on the ground wire and the suspension clamp. The short circuit current is injected from the hanging structure, and then flows to the body and then transmits to the ground wire through the pressing bolts, pressing plate and aluminium armour tape. As the short circuit current is assumed flowing to only one side of the ground wire, the current density on one side of the ground wire is very high, but very low on the other side. The total power loss of the ground wire and suspension clamp is about $59.2 \mathrm{~kW}$, which indicates a total resistance of $296 \mu \Omega$.

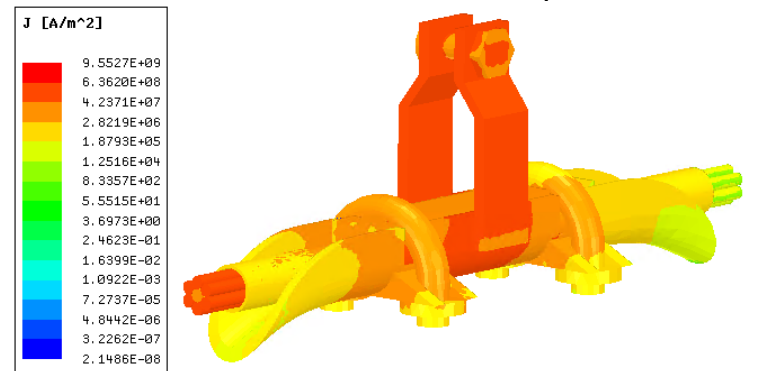

Fig5. Current density distribution on the ground wire and the suspension clamp

Fig. 6 shows the current density distribution on the longitudinal section of the ground wire and the suspension clamp. It shows that the maximum current density occurs at the electrical bridge, which represents the contact point, and the peak current density is as high as $9.6 \times 10^{9} \mathrm{~A} / \mathrm{m}^{2}$. As the current will flow to the end of the ground wire, the conductors on the outer layer of the ground wire has a very high current density. The centre conductor has a low current density due to the bad electrical connection with the outer ones.

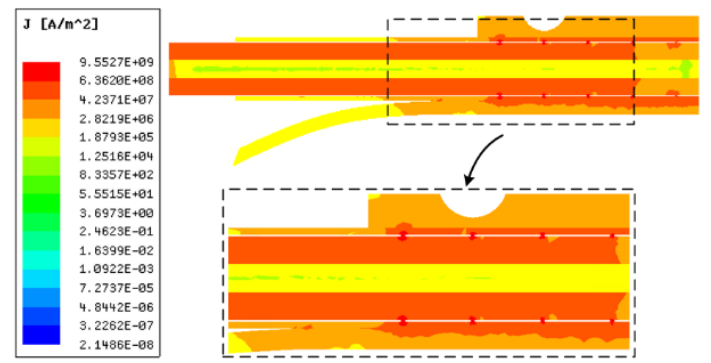

Fig6. Current density distribution on the longitudinal section of the ground wire and the suspension clamp

From Fig. 6, it can be also observed that, the closer to the end of the wire, the higher the current density of the electrical bridge will be. It is also because that the short circuit is assumed flowing to only one end of the round wire. In the conductors of the wire, no skin effect is observed as the current is almost uniformly distributed on the longitudinal section. It can be easily explained by calculating the skin depth of the conductor and compared with the size of the conductor. At the power frequency, the skin depth of the conductor is $d_{\mathrm{s}}=(2 / \mu \omega \sigma)^{1 / 2}=29 \mathrm{~mm}$, which is much larger than the diameter of the conductor. It indicates that, the skin effect is not the primary cause for the breakage of the ground wire.

\subsection{Temperature Rise and Distribution}

In the thermal analysis, the mass density, thermal conductivity and specific heat of the ground wire and suspension clamp is $7750 \mathrm{~kg} / \mathrm{m}^{3}, 50 \mathrm{~W} / \mathrm{m} \cdot \mathrm{K}$ and 480 $\mathrm{J} / \mathrm{kg} \cdot \mathrm{K}$, respectively. For the aluminium armour tape, these values are set $2770 \mathrm{~kg} / \mathrm{m}^{3}, 165 \mathrm{~W} / \mathrm{m} \cdot \mathrm{K}$ and $875 \mathrm{~J} / \mathrm{kg} \cdot \mathrm{K}$, respectively. In addition, the lasting time of the shirt circuit stage is assumed $100 \mathrm{~ms}$, and the initial temperature is assumed $30^{\circ} \mathrm{C}$.

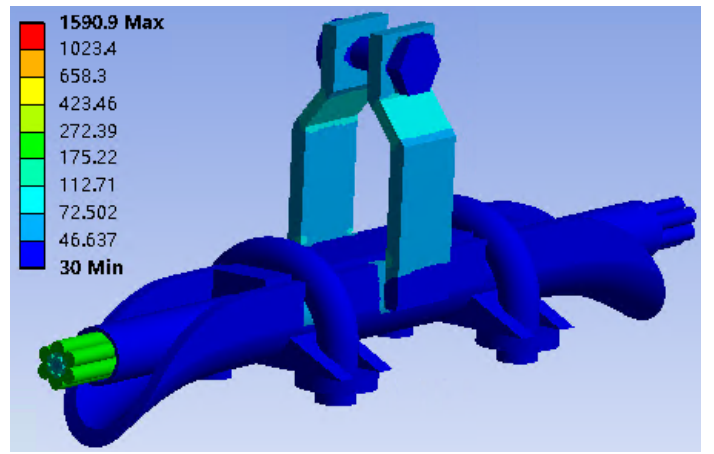

Fig7. Temperature distribution on the ground wire and the suspension clamp@ $\mathrm{t}=100 \mathrm{~ms}$

Fig. 7 shows the temperature distribution on the ground wire and the suspension clamp at the end of the short circuit stage. The maximum temperature is about $1590^{\circ} \mathrm{C}$, which exceeds melting point of ground wire. As the current flows only to only one side of the ground wire, the temperature on one side of the ground wire is much higher than the other side.

Fig. 8 shows the temperature distribution on the longitudinal section of the ground wire and the suspension clamp at the end of the short circuit stage. It shows that the maximum temperature occurs at the electrical bridge, 
which represents the contact point, and the highest temperature exceeds the melting point. As the ground wire should bear the gravity force of itself, breakage will occur under the impact from the gravity force and the rapid temperature rise.

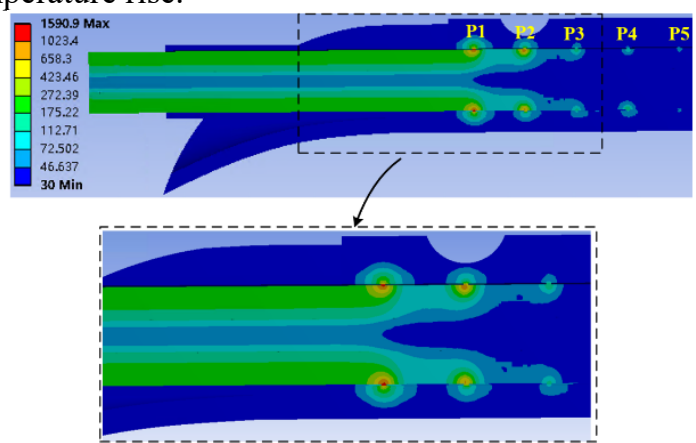

Fig8. Temperature distribution on the longitudinal section of the ground wire and the suspension clamp@ $@ \mathrm{t}=100 \mathrm{~ms}$

Fig. 9 shows the curve of the maximum temperature of the electrical bridges verse time. It shows that, the temperature goes up very rapidly within $20 \mathrm{~ms}$, and then the rising rate slows down. It is due to the thermal conduction from the electrical bridges to the adjacent conductors where the temperature is relatively low. As the temperature difference increases, the thermal conduction will increase, and the temperature rising rate will be reduced.

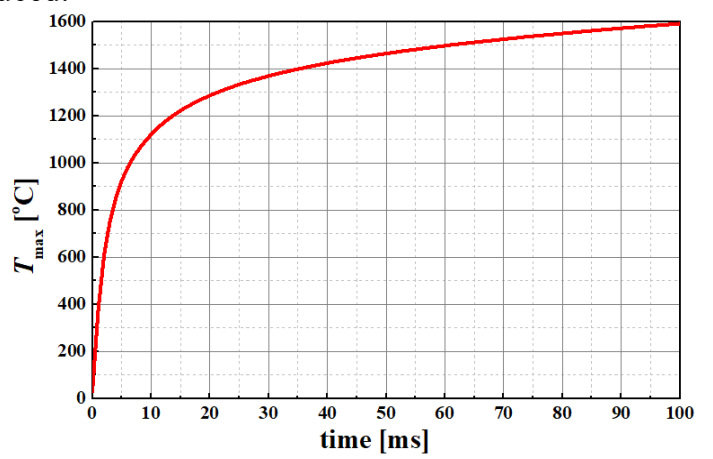

Fig9. Maximum temperature on the electrical brides vs. time

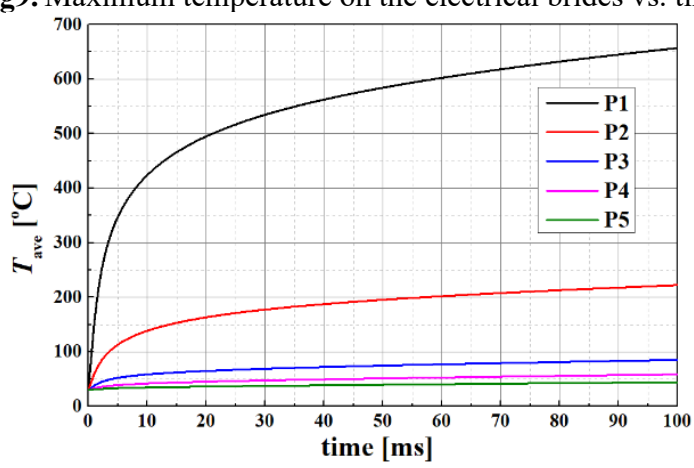

Fig10. Average temperature on the electrical bridges vs. time

Fig. 10 shows the curves of the average temperature of the selected electrical bridges as shown in Fig. 8 verse time. The average temperature is much lower than the maximum value as shown in Fig. 9. The electrical bridge closest to the end of the ground wire has the highest average temperature, which coincides with the analysis results shown in Fig. 6.

\section{Discussions on major factors}

Based on the analysis presented in Section 3, it can be concluded that the temperature rise of the ground wire depends on the contact resistance, the amplitude of the short circuit current and the lasting time of short circuit stage. This section will discuss the influence of these major factors on the temperature rise of the ground wire.

\subsection{Contact Resistance}

In the analysis, the contact points are modelled as electrical bridges with a cylindrical shape. Then the resistance of a contact point is

$$
R_{0}=\frac{\rho h}{\pi r^{2}}
$$

As all the contact points are in parallel, then the total contact resistance of $N_{\mathrm{c}}$ contacts can be expressed as

$$
R=\frac{\eta R_{0}}{N_{c}}=\frac{\eta \rho h}{N_{c} \pi r^{2}}
$$

where $\eta$ is the correction factor as the short circuit current flows through only half of the contact points, and the current density is not uniformly distributed among the contact points. Combining Eq. (1) (2) and (5), the total contact resistance can be expressed as

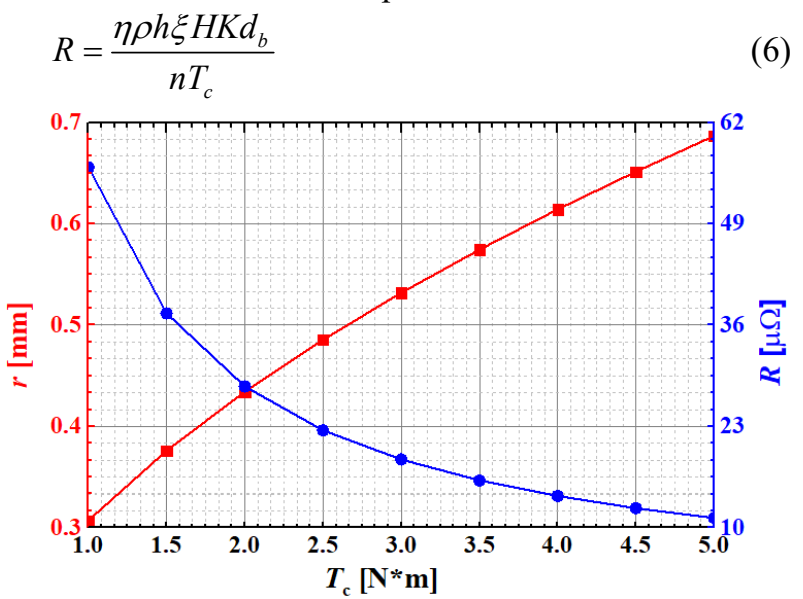

Fig11. Radius of electrical bridge and total contact resistance under different preloaded torques

In Eq. (6), only the preloaded torque on the bolt is easy to be adjusted, so the following will mainly focus on the influence of this factor. Fig. 11 shows the radius of electrical bridge and the total contact resistance under different preloaded torques, where the correction factor $\eta$ is assumed 3. The total contact resistance has an inverse relation with the preloaded torque. When the preloaded torque varies from $1 \mathrm{~N} \cdot \mathrm{m}$ to $5 \mathrm{~N} \cdot \mathrm{m}$, the total contact resistance will decrease from $56.3 \mu \Omega$ to $11.2 \mu \Omega$. Compared with total resistance of the whole system (296 $\left.\mu \Omega @ T_{\mathrm{c}}=5 \mathrm{~N} \cdot \mathrm{m}\right)$, the contact resistance is much small and contributes little to the total power loss.

Fig. 12 shows the maximum temperature and the total power loss of the whole system under different preloaded torques. It shows that, with the increase of the preloaded torque, the total power loss of the whole system decrease only a little. It is because that the contact resistance contributes only a little to the total resistance. However, the increase of the preloaded torque will greatly reduce the 
maximum temperature on the contact points, and thus protect the ground wire from breakage. If the preloaded torque is increased to $5 \mathrm{~N} \cdot \mathrm{m}$, the maximum temperature will decrease to only $378{ }^{\circ} \mathrm{C}$, which is far below the melting point and ensure the reliability of the ground wire.

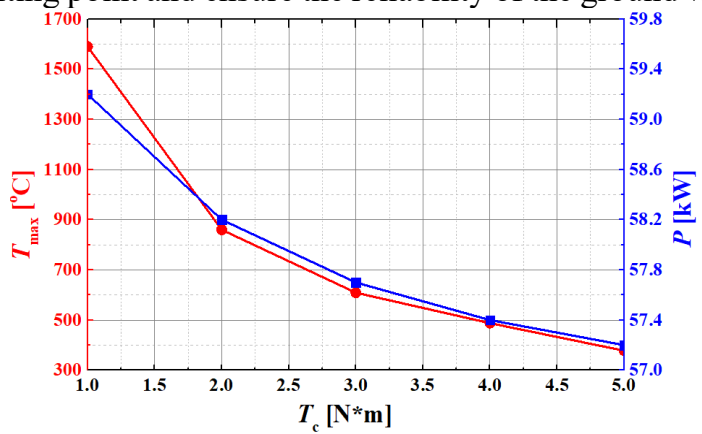

Fig12. Maximum temperature and total power loss of the whole system under different preloaded torques

\subsection{Amplitude of Short Circuit Current}

As the ohmic power loss is in direct proportion to the square of the short circuit current, the amplitude of short circuit current will certainly influence the temperature rise, and thus affect the breakage of the ground wire.

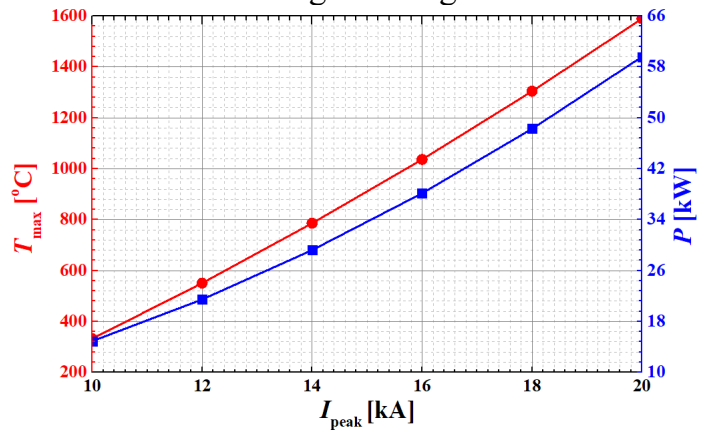

Fig13. Maximum temperature and power loss of the whole system under different amplitudes of short circuit current

Assuming the preloaded torque on the compressing bolts is $1 \mathrm{~N} \cdot \mathrm{m}$, the power loss of the whole model and the corresponding maximum temperature on the contact point are calculated as shown in Fig. 13. As the amplitude of the short circuit current increases, the total power loss will increase in a quadratic manner, and the maximum temperature increases in a similar manner. It indicates that, limiting the amplitude of the short circuit current is very efficient to avoid the breakage of the ground wire.

\subsection{Short Circuit Duration}

When a short circuit occurs, the monitoring system will recognize it and relaying protection system will make an action to cut off the short circuit. However, it takes time to complete the protection procedure. As shown in Fig. 9 and 10 , the temperature rises very quickly during the first $20 \mathrm{~ms}$ of the short circuit duration, and the rising rate will be reduced due to the thermal conduction. This interval during which the temperature rises very quickly is determined by the thermal time-constant of the contact point and adjacent conductors. Reducing the time of the protection procedure will certainly reduce the maximum temperature. However, it is not easy to ensure an action time less than $20 \mathrm{~ms}$. It is also unnecessary as the temperature rises relatively slow during the latter duration.

\section{Conclusions}

This paper presents the breakage mechanism study of overhead ground wire under lighting stoke based on finite element analysis. Analysis results shows that contact resistance effect contribute much more to the breakage than skin effect, and it is the primary cause for the breakage. Further analysis shows that, increasing the preloaded torque on the compressing bolts to reduce the contact resistance is a very efficient measure to reduce the maximum temperature on the contact point during short circuit duration. Besides, limiting the short circuit current is also efficient, but it is not easy to be realized.

\section{References}

1. Z. M. He, W. J. Chen, H. B. Shen. Experimental study of protection measures for preventing $10 \mathrm{kV}$ insulated conductors from breakage caused by lightning stroke. Electricity Distribution - Part 1, 2009. CIRED 2009.

2. Z. Mei, S. M. Chen, et al., Statistic of lighting accidents during 1998-2004 in China, High Voltage Engineering, 33(12), 2007.

3. Yucel, Mehmet K, et al. An ultrasonic guided wave approach for the inspection of overhead transmission line cables. Applied Acoustics 122: 23-34, 2017.

4. Yovanovich, M., and K. Nho. Experimental investigation of heat flow rate and direction on contact resistance of ground/lapped stainless steel interfaces. Thermophysics Conference 2013.

5. Kogut L. Electrical performance of contaminated rough surfaces in contact[J]. Journal of Applied Physics, 97(10): 103723.1-103723.5, 2005.

6. Wang W.G., Liang M.L., et al., Characteristics of static contact resistance between pantograph and catenary, High Voltage Apparatus, 48(1), 2012.

7. Chen Y.M., Song W., Assessment of the uncertainty of the measurement of conductor DC resistance, Electric Wire and Cable, 12(1), 2009.

8. Liu M., A new method for measuring contact resistance, Journal of astronautic metrology and measurement, 25(3), 2005.

9. Laor, A., Herrell, PJ., Mayer, M. A Study on Measuring Contact Resistance of Ball Bonds on Thin Metallization. IEEE Trans. Compon. Packag. Manuf. Technol. 2015, 5, 704-708. 